\title{
Makro- und Mikrokunststoffe in österreichischen Komposten
}

\author{
Christian Zafiu · Erwin Binner · Cornelia Hirsch · Benedikt Vay · Marion Huber-Humer
}

Online publiziert: 7. August 2020

(C) Der/die Autor(en) 2020

\section{Zusammenfassung In dieser Stu- die wurden Makro- und Mikrokunst- stoffe in einem Größenbereich von $10-0,63 \mathrm{~mm}$ in neun Komposten aus drei, nach dem Stand der Technik be- triebenen österreichischen Kompost- \\ Schlüsselwörter Kompost . Mikroplastik · Kunststoffe . Makrokunststoffe \\ Macro- and microplastics in Austrian composts} anlagen untersucht. In den untersuchten Komposten wurden zwischen 13 und 111 Kunststoffpartikel pro kg TM gefunden. Eine Hochrechnung der gefundenen Minimal- und Maximalwerte auf ganz Österreich ergibt jährliche Mikrokunststoff-Emissionen von 0,6 bis $111 \mathrm{~g}$ pro EinwohnerIn, die bei der Kompostierung von biogenen Abfällen vorwiegend durch die Fragmentierung von Störstoffen entstehen. Die Kontamination bestand vor allem aus gängigen Verpackungskunststoffen, wobei der größte Anteil mit bis zu $77 \% \mathrm{TM}$ auf Polyolefine entfiel. Die detaillierte Untersuchung einer Miete während des Kompostierungsprozesses ergab, dass die Partikelzahl und deren Größenverteilung sich während des Rotteprozesses ändern können. Auch jahreszeitlich bedingte Veränderungen des Inputmaterials können einen wesentlichen Einflussfaktor darstellen. Eine Möglichkeit zur Reduktion von Kunststoffpartikeln in Komposten stellt die Endaufbereitung dar, bei der durch unbeabsichtigte Anhaftungsprozesse auch Partikel entfernt werden können, die wesentlich kleiner als die Maschenweite des verwendeten Siebes sind. In der gegenständlichen Untersuchung wurden durch die Absiebung und Endaufbereitung des Kompostes vor allem schwere Partikel entfernt.

Mag. Dr. C. Zafiu (凶) •

DI E. Binner, B.A. · C. Hirsch · B. Vay · Univ.-Prof.in DI Dr. M. Huber-Humer Department für

Wasser-Atmosphäre-Umwelt, Institut für Abfallwirtschaft (ABF-BOKU), Universität für Bodenkultur Wien, Muthgasse 107, 1190 Wien, Österreich christian.zafiu@boku.ac.at
Abstract In this study nine composts from three state-of-the-art composting plants in Austria were investigated for macro- and microplastics in a size range of $10-0.63 \mathrm{~mm}$. In all investigated composts 111-13 plastic particles per $\mathrm{kg}$ DM were found. Transferred to Austria, the data resulted in an annual micro-plastic emissions rate ranging from 0.6 to $111 \mathrm{~g}$ per capita, which were mainly caused by the fragmentation of macroplastic impurities during the composting of biogenic waste. The contamination consisted mainly of common packaging plastics, with polyolefins accounting for the largest share of up to $77 \%$. The investigation of a windrow during composting showed that the particle number and its size distribution can change during the rotting process. Seasonal changes in the input material also show great variations. The greatest reduction was achieved during factory screening, which also removed particles that were much smaller than the mesh size (due to unintended adherence effects), with heavy particles in particular being removed.

Keywords Quality of compost . Microplastic · Impurities · Plastic fragmentation $\cdot$ State-of-the-art composting

\section{Einleitung}

Makro- und Mikrokunststoffe finden sich bereits in weiten Teilen unseres Ökosystems (Ambrosini et al. 2019; Browne et al. 2011; Eerkes-Medrano et al. 2015). Nach den erstmals 1969 dokumentierten Kunststoffen in Mägen von Seevögeln (Kenyon and Kridler 1969) wurden seither Kunststoffpartikel vermehrt auch in anderen Tieren gefunden. Wie eine Untersuchung von Stuhlproben zeigte (Schwabl et al. 2019), nimmt auch der Mensch, beispielsweise über kontaminierte Lebensmittel, Kunststoff auf (Cox et al. 2019). Neben dem aquatischen Ökosystem, das bisher weit intensiver auf Mikrokunststoffe erforscht wurde, finden sich auch erhebliche Kunststoffkontaminationen im terrestrischen Bereich ( $\mathrm{Ng}$ et al. 2018). Diese sind insofern problematisch, da die Mikrokunststoffe über lange Zeiträume im Boden verbleiben und einen großen Teil der Nahrungskette beeinträchtigen könnten. Darüber hinaus zeigen erste Laboruntersuchungen auch unterschiedliche Auswirkungen von Mikrokunststoffen auf die biophysikalischen Eigenschaften der Böden, wie Wasserkapazität, Dichte und mikrobielle Aktivität (Machado et al. 2018) sowie auf das Pflanzenwachstum (Machado et al. 2019).

Unter „Mikroplastik“ versteht man allgemein Kunststoffpartikel mit einem Durchmesser zwischen $5 \mathrm{~mm}$ und $1 \mu \mathrm{m}$ (EFSA 2016), während für „Makroplastik" die Kunststoffe einen Durchmesser $>5 \mathrm{~mm}$ aufweisen müssen. Mikrokunststoffe werden anhand ihrer Herkunft in primäre und sekundäre Mikrokunststoffe eingeteilt (Cole et al. 2011). Primäre "Mikrokunststoffe“ werden Produkten gezielt zugefügt, wie zum Beispiel als Mittel zum Peeling in Kosmetikprodukten. Um den zukünftigen Eintrag an primären Mikrokunststoffen in die Umwelt zu verringern, soll primäres „Mikroplastik“ in Konsumgütern in der EU zukünftig verboten werden. Sekundäres „Mikroplastik“ entsteht durch die Verwitterung (Fragmentierung) von makroskopischen Kunststoffen, die sowohl beim normalen Gebrauch von Kunststoffartikeln (Alterung), als auch durch „Littering“ (unachtsames Wegwerfen) oder unsachgemäße Behandlung (beispielweise durch Verwehungen aus Abfallbehandlungsanlagen) in die Umwelt gelangen. Unabhängig von der Quelle verwittern konventionelle 
Kunststoffpartikel nur sehr langsam und reichern sich daher in der Umwelt an.

In einer Konsortialstudie des Frauenhofer-Instituts Umsicht (Bertling et al. 2018) wurden die Eintragspfade von primären und sekundären Mikrokunststoffen in die Umwelt untersucht. Die Studie kam zu dem Schluss, dass 10\% der Mikrokunststoffkontaminationen in Deutschland der Abfallwirtschaft zuzuschreiben sind und diese somit, nach Reifenabrieb, die zweitgrößte Quelle für den Eintrag von Mikrokunststoffen in die Umwelt darstellt. Die Kompostierung bzw. die Kompostanwendung wurde mit $5,6 \%$ als die größte abfallbedingte Quelle von Mikrokunststoffen in Böden angeführt.

Komposte sind hierbei besonders $\mathrm{zu}$ betrachten, da das Material in der Landwirtschaft eingesetzt wird und somit Mikrokunststoffe in die Nahrungskette gelangen können. Auf der anderen Seite stellt Kompost als Bodenverbesserungsmittel eine wichtige Quelle für Nährstoffe und stabile organische Substanz dar. Durch Kompostierung wurden 2015 in Österreich aus 1,14 Mio t biogenen Abfällen ca. 339.200t Kompost hergestellt und angewendet (BAWP 2017).

Bei der technischen Kompostierung können die in den angelieferten Inputmaterialien enthaltenen Störstoffe, wie z.B. Tragetaschen, durch mechanische, biochemische und thermische Beanspruchung zu MikrokunststoffPartikeln fragmentiert werden (Andrady 2017; Qi et al. 2020). Neben dieser Quelle kann das Inputmaterial bereits eine „Mikroplastik“-Grundbelastung aufweisen. Auch ein atmosphärischer Eintrag von mikroskopisch kleinen Partikeln, z.B. aus dem Straßenverkehr, ist möglich. Die angeführten Eintragsquellen (Luft, Fehlwürfe oder primäres „Mikroplastik“) machen klar, dass der Kompostanlagenbetreiber wenig Möglichkeiten zur Verringerung des Mikrokunststoffanteils im Endprodukt hat. Die einzige Möglichkeit stellt die mechanische Abtrennung von Störstoffen aus den angelieferten Bioabfällen dar, die allerdings wegen des hohen Wassergehalts in der Regel nur wenig effizient ist. Die deutlich effektivere manuelle Aussortierung wird aus hygienischen Gründen meist abgelehnt. Die einzige reelle Möglichkeit zur Verringerung der Mikrokunststoffbelastung in Komposten (wie auch generell in der Umwelt) stellt daher die Vermeidung von

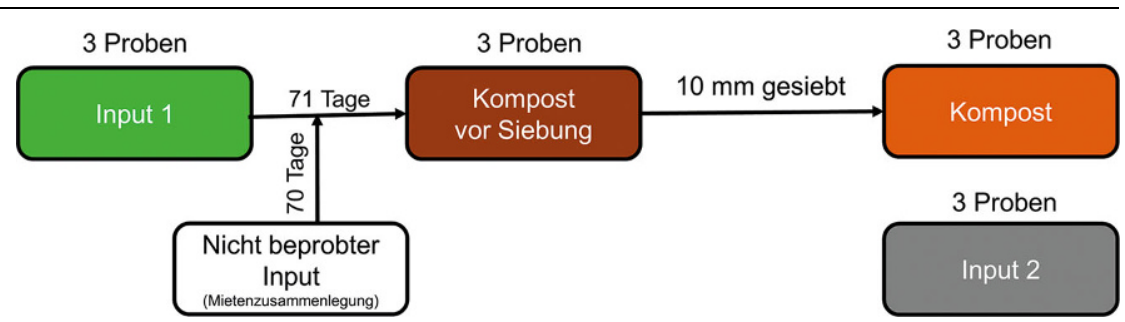

Abb. 1 Prozessabhängige Probenahme in Kompostanlage A

konventionellen Kunststoffen (Substitution) vor allem in Einwegprodukten dar.

Im Rahmen dieser Studie wurden neun Kompostproben aus drei österreichischen Kompostanlagen auf Kunststoffpartikel im Größenbereich zwischen 10 und $0,63 \mathrm{~mm}$ untersucht. Ziel der Untersuchung war, die Variabilität der Partikelanzahl und Masse zu erfassen. In einer weiteren Untersuchung wurde eine Miete in verschiedenen Prozessabschnitten beprobt, beginnend beim Aufsetzen der Miete (Input), unmittelbar vor der Werksabsiebung (Rottegut) und unmittelbar danach (Kompost). Damit konnten prozessbedingte Veränderungen beurteilt werden.

\section{Material und Methoden}

Die in dieser Studie untersuchten Proben stammen aus drei österreichischen, nach Stand der Technik betriebenen Kompostanlagen.

In Anlage A wird Biotonnematerial mit hohem Anteil an Baum- und Strauchschnitt in natürlich belüfteten Dreiecksmieten kompostiert. Die Mieten werden mehrmals wöchentlich umgesetzt und bei Bedarf befeuchtet. Nach 6 bis 10 Wochen Rottedauer wird das Rottegut mittels Trommelsiebes (10 mm) abgesiebt.

In den Anlagen B und C wurde eine Mischung von Biotonnematerial, Baum- und Strauchschnitt sowie Siebresten als Strukturmaterial und Bodenaushub (Anteil ca. 7\%) in Dreiecksmieten kompostiert. Anlage B arbeitet mit schwach drückender Zwangsbelüftung (wöchentliches Umsetzen während der Intensivrotte), Anlage $\mathrm{C}$ mit natürlicher Belüftung (tägliches Umsetzen während der Intensivrotte). Bei Bedarf wurde das Rottegut während des Umsetzens befeuchtet. Nach einer Rottedauer von jeweils 12 Wochen wurde das Rottegut mittels Trommelsiebes $(10 \mathrm{~mm})$ abgesiebt.

\subsection{Probenahme von Rottegut während des Kompostierungsprozesses in Anlage A}

Um die prozessbedingten Veränderungen des Mikrokunststoffanteils im Substrat zu untersuchen, wurden aus einer Miete von Anlage A jeweils 3 Proben $\mathrm{zu}$ unterschiedlichen Zeitpunkten der technischen Kompostierung entnommen (Abb. 1). Die 3 Inputproben (Input 1) von jeweils ca. $20 \mathrm{~kg}$ Feuchtmasse (FM) wurden unmittelbar nach dem Aufsetzen (November 2019) entnommen.

Da in Anlage A nach ca. 1 Woche Rottedauer jeweils im Routinebetrieb 2 Mieten zu einer vereinigt wurden (in der Versuchsplanung nicht berücksichtigt), enthielten die weiteren Proben nicht nur Material der beprobten Miete (Input 1) sondern auch Material einer weiteren nicht beprobten Inputcharge. Nach 10 Wochen Rottedauer erfolgte im Jänner 2020 die Werksabsiebung mit zwei hintereinander angeordneten mobilen 10-mm-Trommelsieben. Während des Siebvorganges wurden in 30minütigen Abständen je 3 Rottegutproben (ca. $15 \mathrm{~kg}$ FM „Vor Sieb“) vor der Siebung und die jeweils „korrespondierenden" Proben vom Siebdurchgang $<10 \mathrm{~mm}$ (ca. $10 \mathrm{~kg} \mathrm{FM} \mathrm{„Kompost“)}$ entnommen. Die Proben vom Siebdurchgang werden in diesem Artikel beim Vergleich der Komposte als „Kompost 7" bezeichnet.

Um eine erste Abschätzung der Varianz der Kunststoffkontamination im Inputmaterial durchzuführen, wurden am Tag der Werksabsiebung auch Proben einer weiteren neu aufgesetzten Miete entnommen („Input 2“).

\subsection{Probenahme von Komposten aus Nachrottephase}

In Anlage A wurden zudem Kompostproben von jeweils ca. $20 \mathrm{~kg}$ FM von sechs in der Nachrotte befindlichen Mieten entnommen. Die Rottedauer betrug 7 bis 11 Wochen. Die ent- 
sprechenden Inputmaterialien stammen aus dem Zeitraum 29.05.2019 bis 27.06.2019. Die Probenahme erfolgte nach dem Abtragen der äußeren $50 \mathrm{~cm}$ aus dem Kernbereich der Nachrottemieten. Die Komposte erhielten die Bezeichnungen 1 bis 6 . Die Kompostprobe 7 (Rottedauer 10 Wochen) entspricht der in 2.1. beschriebenen Probe „Nach Sieb“.

In den Anlagen B wurden sechs Proben und in Anlage $\mathrm{C}$ vier Proben aus jeweils einem in der Nachrotte (Rottedauer 12 Wochen) befindlichen Kompost nach Werkssiebung $<10 \mathrm{~mm}$ entnommen und untersucht.

\subsection{Fraktionierung von Rottegut- und Kompostproben}

Die Proben „Input 1“ und „Kompost Vor Sieb“ wurden im Labor mithilfe eines Labortrommelsiebes in Fraktionen $>10 \mathrm{~mm}$ und $<10 \mathrm{~mm}$ getrennt. Kunststoffpartikelanzahl und Masse aus der Fraktion $>10 \mathrm{~mm}$ wurden auf Trockenmasse (TM) der Gesamtprobe bezogen. Jeweils ca. 100 g FM der Kompostproben und der Fraktionen $<10 \mathrm{~mm}$ der Proben „Input 1“ und „Vor Sieb“ wurden mittels Nasssiebung (Siebturm AS 200, Retsch) feucht in die Fraktionen $10-6,3 \mathrm{~mm}, 6,3-2 \mathrm{~mm}$ und $2-0,63 \mathrm{~mm}$ separiert. Parallel wurde an einer weiteren Probe der jeweiligen Fraktion $<10 \mathrm{~mm}$ der Wassergehalt bei $105^{\circ} \mathrm{C}$ bestimmt. Die sich daraus ergebenen Trockenmassen der Einzelstichproben sind in Tab. 1 angegeben. Während der Siebung wurden die Proben mit Leitungswasser gespült, bis das Waschwasser klar wurde (Dauer ca. 10-15 min).

Tab. 1 Bezeichnung und Stichprobengröße der Komposte für die Bestimmung des Mikrokunststoffanteils. DieStichprobenmasse von Kompost A 7, B und C ist der Mittelwert von Mehrfachbestimmungen

\begin{tabular}{|l|l|}
\hline Bezeichnung & Stichprobe [g TM] \\
\hline Kompost A 1 & 67,4 \\
\hline Kompost A 2 & 66,1 \\
\hline Kompost A 3 & 71,7 \\
\hline Kompost A 4 & 70,2 \\
\hline Kompost A 5 & 78,1 \\
\hline Kompost A 6 & 63,1 \\
\hline Kompost A 7 & $36,7\left({ }^{\star} \pm 0,7\right)$ \\
\hline Kompost B & $59,3\left({ }^{*} \pm 1,4\right)$ \\
\hline Kompost C & $94,2\left({ }^{*} \pm 3,1\right)$ \\
\hline
\end{tabular}

(mit * in Klammer ist die Standardabweichung angegeben)
Die Fraktionen wurden in Bechergläser überführt und bei $105^{\circ} \mathrm{C}$ über Nacht getrocknet um die TM zu bestimmen. Potenzielle Kunststoffe aus den Fraktionen $>10 \mathrm{~mm}, 10-6,3 \mathrm{~mm}$ und $6,3-2 \mathrm{~mm}$ wurden mit freiem Auge von der Matrix separiert. Potenzielle Kunststoffe aus der Fraktion 2-0,63 mm wurden unter einem Stereomikroskop (SZX-ILLD 200, Olympus Optical) getrennt.

Aufgrund der Verwendung des in der Kompostanalyse üblichen Mesh-Siebsatzes wurde die Grenze zwischen Makro- und Mikrokunststoffen mit 6,3 mm und nicht wie von der EFSA (2016) vorgeschlagen bei $5,0 \mathrm{~mm}$ gewählt. Partikel in der Kunststofffraktion 6,3-2 mm wurden allerdings von Hand auf einen Durchmesser $<5 \mathrm{~mm}$ geprüft, mit dem Ergebnis, dass alle gefundenen Kunststoffpartikel einen maximalen Durchmesser von $5 \mathrm{~mm}$ unterschritten und somit den Mikrokunststoffen gemäß EFSA zuzuordnen sind.

\subsection{Bestimmung der Kunststoffart}

Potenzielle Kunststoffe aus den Fraktionen wurden mittels Diamant Augmented Total Reflection Fourier Transform Infrared (ATR-FTIR)-Spektrometer (Bruker Alpha,), das mit einem DTGS (Dotierter-Triglycinsulfat)-Detektor ausgestattet ist, untersucht. Jedes Spektrum wurde als Mittelwert aus 24 Wellenlängenscans in einem Bereich von 4000 bis $500 \mathrm{~cm}^{-1}$ mit einer Auflösung von $4 \mathrm{~cm}^{-1}$ errechnet. Überlagernde Wasserdampf- und $\mathrm{CO}_{2}$ Banden wurden durch die Softwarefunktion „Atmosphärische Kompensation" herausgefiltert und die resultierenden Spektren wurden vektornormiert. Die gefilterten und normierten Spektren wurden mit der Biorad-SpektrenBibliothek (konventionelle Kunststoffe) sowie einer institutseigenen Spektren-Bibliothek (biologisch abbaubare Kunststoffe) verglichen. Um als Kunststoff klassifiziert zu werden, mussten die Probenspektren zumindest zu 50\% mit dem entsprechenden Referenzspektrum übereinstimmen. Darüber hinaus wurden die Spektren auch qualitativ verglichen, um etwaige Fehlinterpretationen des automatisierten Vergleichs zu vermeiden. Erst wenn der Abgleich durch die Datenbank eine Übereinstimmung von $>50 \%$ ergab und die qualitative, händische Überprüfung des Bandenmusters positiv war, wurde ein „potenzielles“ Kunststoffpartikel als „tatsächlicher“ Kunststoff gewer- tet. Gewertete Kunststoffe wurden auf einer Analysewaage (GR-202, A \& D Instruments Ltd.) gewogen (Genauigkeit: $0,1 \mathrm{mg}$ ).

\section{Ergebnisse und Diskussion}

3.1 Veränderung der Makro- und Mikrokunststoffbelastung während der technischen Kompostierung

Um das Ausmaß der Kunststoffkontaminationen zu Beginn und die Veränderung während der Prozesse in einer Kompostanlage $\mathrm{zu}$ untersuchen, wurden wie erwähnt Proben zu unterschiedlichen Zeitpunkten (wie in Abb. 1 gezeigt) entnommen, aufbereitet und analysiert.

Inputproben und die Proben vor der Siebung wurden zunächst durch ein 10 mm-Labortrommelsieb vorfraktioniert. Die enthaltenen Kunststoffe $>10 \mathrm{~mm}$ wurden manuell aussortiert und bewertet. Fraktionen $<10 \mathrm{~mm}$ dieser Proben und jene aus der Werkssiebung wurden mittels Siebturms fraktioniert. Alle Proben „Nach Sieb“ (= Kompost 7) wiesen keine Kunststoffe in der Fraktion $>10 \mathrm{~mm}$ auf, da diese bereits durch die Werksabsiebung entfernt worden waren. In Abb. 2 sind die Ergebnisse der Fraktionierung dargestellt. Diese zeigen, dass sich während des Rotteprozesses (von „Input 1“ zu „Vor Sieb“) die Anzahl an Kunststoffpartikeln in den betrachteten Größenbereichen insgesamt um $15 \%$ reduzierte. Es fanden allerdings in den einzelnen Fraktionen sehr unterschiedliche Veränderungen statt. Die Anzahl an Kunststoffpartikeln in der Fraktion $>10 \mathrm{~mm}$ erhöhte sich um $96 \%, 10-6,3 \mathrm{~mm}$ hingegen nahm nur um $12 \%$ zu, während in der Fraktion $6,3-2 \mathrm{~mm}$ eine Zunahme von $204 \%$ gefunden wurde. Die kleinste Fraktion $(2-0,63 \mathrm{~mm})$ wies eine Abnahme der Partikelzahl um $60 \%$ auf und war für die Gesamtreduktion von $15 \%$ verantwortlich. Da die Fraktionen sowohl Zuals auch Abnahmen aufweisen, ist eine gesamtheitliche Interpretation derzeit schwierig. In den jeweiligen Fraktionen kann ein relativer Anstieg der Anzahl an Partikeln durch den allgemeinen Masseverlust (Rotteverlust bzw. durch das Ausschleusen des Siebüberlaufs) erklärt werden, während Abnahmen in größeren und gleichzeitige Zunahmen in kleineren Fraktionen durch die Fragmentierung der Kunststoffe und den Transfer in kleinere Fraktionen erklärbar sind (Abb. 3). 


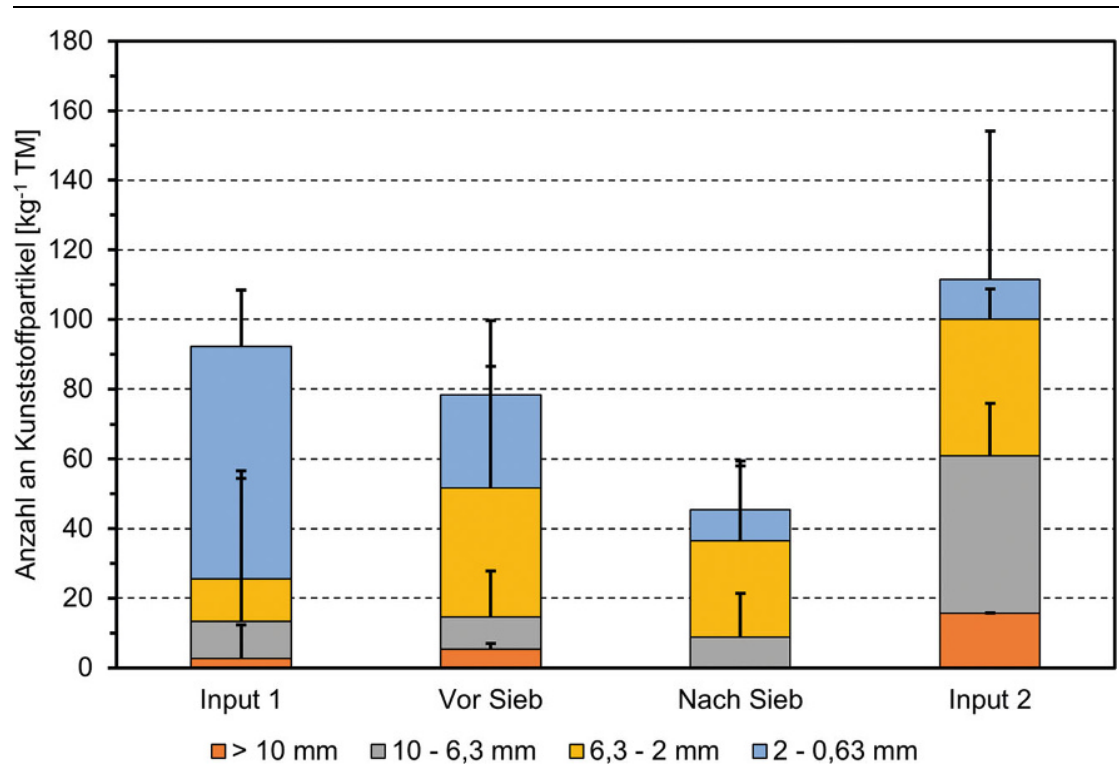

Abb. 2 Untersuchung von Rottegut auf die Anzahl an Kunststoffpartikel (in Stück pro $\mathrm{kg}$ Trockenmasse). Untersucht wurden Input 1 (November 2019), das daraus hergestellte Rottegut nach 10 Wochen Rottedauer vor dem Sieben („Vor Sieb“) und dem Kompost $10 \mathrm{~mm}$ („Nach Sieb“) sowie einemam Tag der Absiebung angelieferten Input2 (Jänner 2020). Die Probe „Nach Sieb“ entspricht dem Kompost 7 in 3.2. Es wurden jeweils drei Proben entnommen, die als Mittelwerte dargestelltwerden, wobei die Fehlerbalken den Standardabweichungen entsprechen

Die Werksiebung bei $10 \mathrm{~mm}$ („nach Sieb“) reduzierte die gesamte Zahl der Kunststoffpartikel um $42 \%$ gegenüber den Proben vor diesem Prozessschritt („Vor Sieb“). Nach Fraktionen betrachtet, werden, wie zu erwarten, in der Fraktion $>10 \mathrm{~mm}$ Kunststoffpartikel deutlich reduziert (um 100\%), da alle Partikel $>10 \mathrm{~mm}$ durch die Absiebung effizient entfernt wurden. Kunststoffpartikel in der Fraktion 10-6,3 mm wurden durch die Siebung nur um 5\% reduziert, während $6,3-2 \mathrm{~mm}$ um $25 \%$ und die Fraktion 2-0,63 mm um $67 \%$ reduziert wurden. Dieses Ergebnis ist interessant, da Kunststoffpartikel vor allem in den feineren Fraktionen reduziert wurden, die eigentlich das Sieb hätten passieren können und daher in der Probe gefunden werden sollten. Dieses Ergebnis kann derzeit nicht eindeutig interpretiert werden und lässt mehrere Erklärungsmöglichkeiten offen. Eine Erklärung wäre, dass kleine Kunststoffpartikel an großen Matrixkomponenten oder Störstoffen haften bleiben und damit im Siebüberlauf landen. Dies könnte vor allem bei höheren Feuchtegehalten des Rotteguts eine Rolle spielen. Die zweite Erklärungsmöglichkeit wäre, dass die eingesetzten Trommelsiebe größere Kunststoffpartikel fragmentieren. Diese Fragmentie- rung müsste jedoch zu derart kleinen Mikrokunststoffpartikeln erfolgen, dass diese unterhalb der Nachweisgrenze liegen $(<0,63 \mathrm{~mm})$.

Um Schwankungen der Kunststoffkontaminationen in angeliefertem Rottegutmaterial grob abschätzen zu können, wurde neben Input 1 (November 2019) am Tag der Werkssiebung eine weitere Inputprobe, Input 2 (Jänner 2020), entnommen. Abb. 2 zeigt, dass sowohl Anzahl an Kunststoffpartikeln als auch die Größenverteilung zwischen den beiden Inputmaterialien stark variieren. Die Gesamtzahl der Partikel im Input 1 war dabei um $17 \%$ geringer als in Input 2. Der Fraktionsvergleich weist eine deutliche Heterogenität in der Partikelgrößenverteilung der beiden Inputmaterialien auf. Die drei gröberen Fraktionen von Input 1 weisen deutlich geringere Kunststoffpartikelanzahlen auf als Input 2: $>10 \mathrm{~mm}(-83 \%)$, $10-6,3 \mathrm{~mm}$ (-77\%), 6,3-2 mm (-69\%), während bei der feinsten untersuchten Fraktion $(2 \mathrm{~mm}-0,63 \mathrm{~mm})+479 \% \mathrm{mehr}$ Kunststoffpartikel in Input 1 gefunden wurden. Diese Ergebnisse zeigen die große Variationsbreite der angelieferten Inputmaterialien. Da die Mieten jeweils an den Tagen der Probenahmen aufgesetzt wurden, kann eine nachträgliche Kontamination (z. B. Windver- frachtung) weitgehend ausgeschlossen werden. Der Grund ist möglicherweise jahreszeitbedingt. Der geringer kontaminierte Input 1 stammt aus dem Herbst, der einen sehr hohen (verdünnend wirkenden) Laubanteil aufwies. Andererseits könnte auch eine erhöhte Kunststoffkontamination des Strukturmaterials, das für Input 2 (10 Wochen später angeliefert) verwendet wurde, für die höheren Werte verantwortlich sein.

Abb. 4 zeigt die Ergebnisse der Analysen der einzelnen Kunststoffpartikelmassen für das Rottegut vor und nach der Werkssiebung bei $10 \mathrm{~mm}$. Diese Analyse zeigt, dass die gefundenen Partikelmassen durch die Werkssiebung um $95 \%$ reduziert wurden. Nach Fraktionen betrachtet erfolgt, wie $\mathrm{zu}$ erwarten, eine $100 \%$ ige Reduktion für Kunststoffe $>10 \mathrm{~mm}$. In dieser Fraktion fanden sich vor dem Siebvorgang $65 \%$ der Kunststoffe. Auch die Fraktionen $10-6,3 \mathrm{~mm}(-92 \%)$ und 6,3-2 mm $(-85 \%)$ wiesen eine starke Reduktion auf, während die Fraktion 2-0,63 mm immer noch eine Massereduktion von $-67 \%$ aufwies. Überraschend ist, dass die Reduktion der Massen stärker ausfiel als jene der Partikelanzahlen. Das bedeutet, dass vor allem schwere bzw. (auf die Größenfraktion betrachtet) dichte Kunststoffpartikel während der Werkssiebung entfernt wurden.

Der Quotient Masse/Anzahl der Kunststoffpartikel für die untersuchten Fraktionen erlaubt eine Abschätzung der Reduktion der Kunststoffpartikelmassen durch die Werksabsiebung. Wenn man nun den Quotienten $\left(\mathrm{m}_{\mathrm{M}}=\mathrm{m} / n\right)$ aus der gesamten Partikelmasse (m) und Partikelanzahl (n) und für jede Fraktion bildet, erhält man die mittleren Partikelmassen $\left(\mathrm{m}_{\mathrm{M}}\right)$ aller gefunden Partikel in der jeweiligen Fraktion, die eine Überprüfung und die Berechnung des Ausmaßes der Reduktion der mittleren Partikel als Quotient der Partikelmassen nach und vor der Werkssiebung zulässt (NS/VS). Die Quotienten in Tab. 2 zeigen, dass die Partikelmassen für das Rottegut vor dem Sieben mit der Partikelgröße abnehmen, während jene nach der Siebung in allen Fraktionen Werte unter $2,9 \mathrm{mg}$ pro $\mathrm{kg} \mathrm{TM}$ aufweisen. In der Fraktion $10-6,3 \mathrm{~mm}$ beträgt die mittlere Partikelmasse nach dem Sieben nur noch $8 \%$ und zwischen $6,3-2 \mathrm{~mm}$ $21 \%$ der Partikelmasse vor dem Sieben, während die kleinste betrachtete Fraktion unverändert bleibt. Das Ergeb- 
a
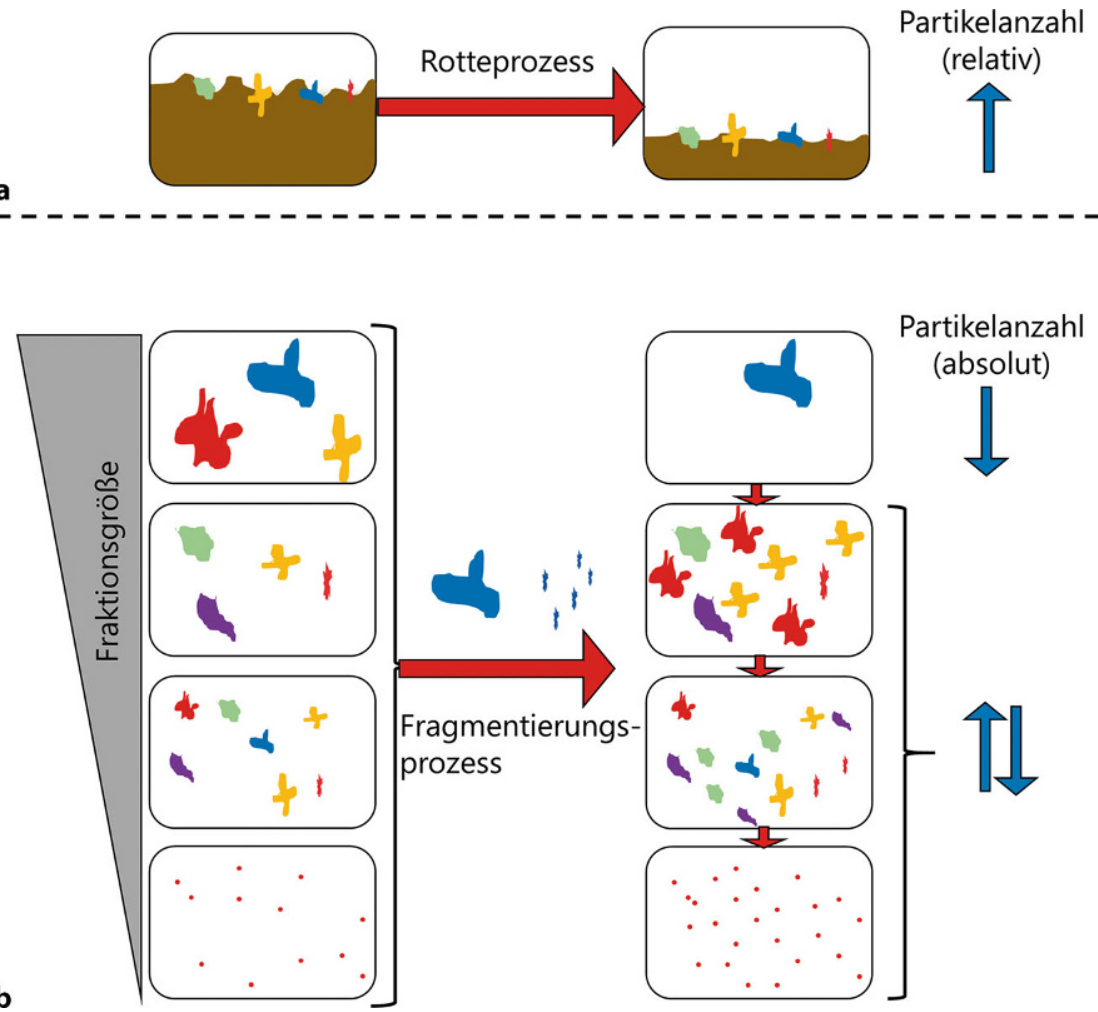

Abb. 3 Schematische Darstellung der Mechanismen, die zu einer Veränderung der Anzahl an Kunststoffpartikeln in den Siebfraktionen führen können. a Die Darstellung beschreibt die relative Zunahme der Partikelanzahl aufgrund der Abnahme der organischen Matrix durch die biologischen Abbauprozesse während der Kompostierung. b Die Darstellung zeigt die Veränderungen der Partikelanzahl in unterschiedlichen Fraktionen. Diegröbste untersuchteFraktionerleidetimmer einen Verlust durch Fragmentierung von Kunststoffen, die in feineren Fraktionen aufgefangen werden. In den mittleren Fraktionen kann es sowohl zu absoluten $\mathrm{Zu}$ - als auch Abnahmen kommen

nis zeigt, dass in den großen Fraktionen (10-2 mm) durch die Werkssiebung mehrheitlich schwere (dichte) Partikel abgesondert werden.

Die Art der gefundenen Kunststoffe wurde durch ATR-FTIR-Messungen mit anschließendem Abgleich mit Spektrendatenbanken durchgeführt und identifizierte insgesamt 9 Polymerarten in den Proben. Die größte Heterogenität wiesen dabei die Proben $>10 \mathrm{~mm}$ auf (Abb. 5), in denen die höchsten Partikelzahlen gefunden wurden. Im Input 1 wurden vorwiegend PA (Polyamid, 42\%), sowie PET (Polyethylenterephtalat, $26 \%$ ), PP/EDPM und PP (Polypropylen/Ethylen Propylen Dien Kautschuk, zusammen $23 \%$ ) und gerin-

Tab. 2 Quotienten Kunststoffpartikelmasse $\left(m_{M}=m / n\right) z u$ Anzahl und deren Verhältnisse (NS/VS) nach und vor Werkssiebung (Mittelwerte aus jeweils 3 Proben) in den untersuchten Fraktionen gere Anteile an PC/PBT (Polycarbonat/ Polybutylterephtalat, 5\%) und HDPE (High Density Polyethylen, 4\%) detektiert. Interessanterweise wurde kein LDPE (Low Density Polyethylen) gefunden, obwohl dieser Kunststoff in der Probe „Vor Sieb“ mit 52\% der häufigste vorgefundene Kunststoff in der Fraktion $>10 \mathrm{~mm}$ war. Dieses Ergebnis deutet darauf hin, dass die Inputproben (Input 1) die Kompostproben („Nach Sieb“) hinsichtlich ihrer Zusammensetzung nicht gut abbilden, bzw. durch Zusammenlegen mit der nicht beprobten Miete (siehe Abb. 1 „nicht beprobter Input“) verändert wurde. Im Vergleich dazu weist Input 2 eine ähnliche Zusammensetzung wie „Vor Sieb“ auf, ob- wohl diese Proben nicht direkt zusammenhängen. Input 2 könnte demnach möglicherweise eine repräsentativere Kunststoffzusammensetzung abbilden als Input 1. Eine mögliche Erklärung ist, dass der hohe Laubanteil in Input 1 aus dem Herbst 2019 die Kunststoffpartikelanzahl, die vermutlich vorwiegend über die Biotonne eingetragen wird, „verdünnte“ und dadurch der relative Anteil im selben Probenvolumen geringer war. Diese Annahme müsste durch entsprechende verdichtete Untersuchungen über ein gesamtes Jahr überprüft werden.

Sowohl in Input 2, wie auch im Rottegut „Vor Sieb“ dominierte LDPE, das zusammen mit HDPE jeweils $62 \%$ aller Kunststoffpartikel ausmachte. Große Anteile wiesen noch PP und PP/EDPM (zusammen $14 \%$ ) in Input 2 und im Rottegut „Vor Sieb“ auf. PET wurde zu $15 \%$ in Input 2 und $7 \%$ im Rottegut „Vor Sieb“ gefunden. Interessanterweise wiesen sowohl Input 2 als auch das Rottegut „Vor Sieb“ geringe Mengen an ABS (Acrylnitril Butadien Styrol) sowie HIPS (High Impact Polystyrol) auf, die häufig in Elektrogeräten eingesetzt werden. In Input 2 wurden bereits $6 \%$ biologisch abbaubare Kunststoffe gefunden, was darauf hindeutet, dass der Ersatz von konventionellen Knotenbeutel durch solche aus biologisch abbaubaren Kunststoffen durch den Handel bereits im Inputmaterial der Kompostanlage abgebildet wird.

Die gefundenen Kunststoffarten in den Proben Input 1, Rottegut „Vor Sieb“, Kompost „Nach Sieb“ und Input 2 für die jeweils untersuchten Fraktionen zwischen 10 und $0,63 \mathrm{~mm}$ sind in Abb. 6 dargestellt. Für die Fraktion $10-6,3 \mathrm{~mm}$ zeigt sich, dass in den ,voneinander abhängigen“ Proben Input 1, „Vor Sieb“ und „Nach Sieb“, jeweils nur ein Partikel (unterschiedlicher Art) gefunden wurde. In Input 1 wurde hierbei LDPE gefunden, das in der Fraktion $>10 \mathrm{~mm}$ nicht gefunden wurde (Abb. 5), obwohl es im Allgemeinen die häufigste Kunststoffart ist. In „Vor Sieb“ wurde 1 Partikel PP gefunden und in „Nach Sieb“ 1 Partikel LDPE. Input 2 beinhaltete verschiedene Kunststoffarten von denen zu jeweils $25 \%$ bioabbaubare

\begin{tabular}{|c|c|c|c|}
\hline Fraktion & $\begin{array}{l}\text { Vor Sieb (VS) } \\
{[\mathrm{mg}]}\end{array}$ & $\begin{array}{l}\text { Nach Sieb (NS) } \\
\text { [mg] }\end{array}$ & NS/VS [\%] \\
\hline $10-6,3 \mathrm{~mm}$ & 16,8 & 1,4 & 8 \\
\hline $6,3-2 \mathrm{~mm}$ & 14,1 & 2,9 & 21 \\
\hline $2-0,63 \mathrm{~mm}$ & 0,1 & 0,1 & 100 \\
\hline
\end{tabular}




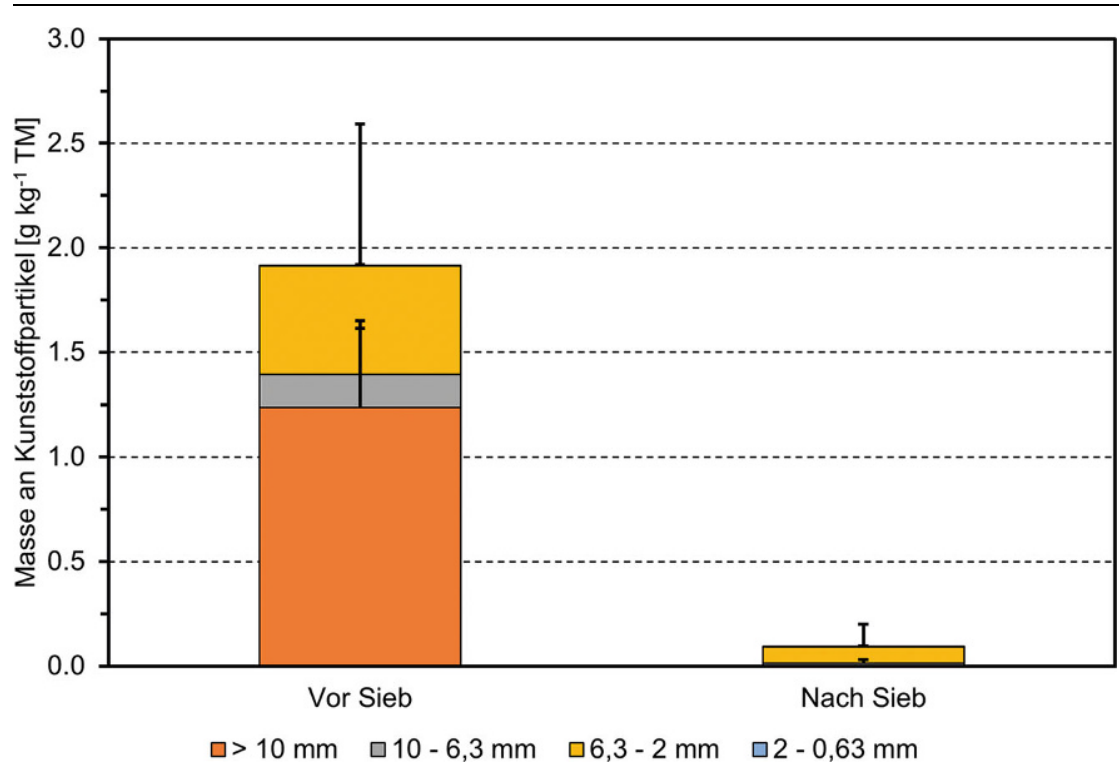

Abb. 4 Untersuchung von Rottegut aus der Anlage A auf die Kunststoffpartikelmasse (in g pro kg Trockenmasse). Untersucht wurden das Rottegut vor dem Sieben („,Vor Sieb“) und der Kompost $<10 \mathrm{~mm}$ (,Nach Sieb“). Dargestellt sind die anteiligen Kunststoffmassen der untersuchten Partikelgrößen. Es wurden jeweils drei Proben entnommen, die als Mittelwerte darstellt werden, wobei die Fehlerbalken den Standardabweichungen entsprechen

Kunststoffe, LDPE, PA, sowie PP und PP/EDPM (zusammen) gefunden wurden. In der Fraktion 6,3-2 mm wiesen die Inputs die höchste Heterogenität auf. In Input 1 befanden sich 4 Kunststoffarten, von denen PVC (Polyvinylchlorid) und PA mit einer Häufigkeit von jeweils $33 \%$ und PP und POM (Polyoxymethylen) mit einem Anteil von jeweils $17 \%$ vorkamen. In Input 2 wurden LDPE (43\%), PA (29\%) sowie PET und PP mit jeweils $14 \%$ gefunden. Die Probe „Vor Sieb“ enthielt zu 75\% LDPE sowie $25 \%$ PU (Polyurethan), während nach dem Sieben LDPE, PU und PET $\mathrm{zu}$ jeweils $33 \%$ gefunden wurden. In der kleinsten untersuchten Fraktion bei PP und PP/EDPM zusammen $58 \%$ ausmachten und LDPE, PA und PVC $\mathrm{zu}$ jeweils $14 \%$ vorzufinden waren. In "Vor Sieb“ wurden PU (50\%) und abbaubare Kunststoffe (50\%) gefunden, während die Probe „Nach Sieb“ nur beinhaltete. In Input 2 wurden PVC und PET zu jeweils $50 \%$ gefunden.
3.2 Makro- und Mikrokunststoffe in Komposten aus der Nachrotte

Insgesamt wurden sieben Komposte aus Anlage A und jeweils ein Kompost aus Anlage B und C auf Makro- und Mikrokunststoffe in den Fraktionen $10-6,3 \mathrm{~mm}, 6,3-2 \mathrm{~mm}$ und $2-0,63 \mathrm{~mm}$ untersucht. Die Ergebnisse der Kunststoffanalyse nach Partikelanzahl in Abb. 7 zeigen schwankende Partikelzahlen im gesamten Größenbereich (10-6,3 mm) mit minimal 13 (Kompost aus Anlage C) und maximal 111 (Kompost 6 aus Anlage A) Partikel pro kg TM. Im Mittel finden sich im Kompost der Anlage A $69( \pm 29)$ Kunststoffpartikel pro kg TM. Der Median mit 57 Partikeln (Kompost 4 aus Anlage A) weist allerdings darauf hin, dass nur wenige der untersuchten 9 Komposte hohe Partikelzahlen beinhalten. Ein weiteres Ergebnis ist, dass nicht in jeder Kornfraktion der Stichproben Kunststoffe gefunden wurden. Das weist wiederum auf eine heterogene Größenverteilung hin, wobei die Auswertung der Mittelwerte der Fraktionen einen Anstieg hin zu höheren Partikelzahlen in den feineren Fraktionen zeigen: $9(10-6,3 \mathrm{~mm})$, $24(6,3-2 \mathrm{~mm})$ und $37(2-0,63 \mathrm{~mm})$ Partikel pro kg TM in den jeweiligen Fraktionen. Eine derartige Zunahme mit feinerer Korngröße ist erklärbar, da während des Kompostierungsprozesses Kunststoffe immer weiter fragmentiert werden und sich dadurch die Stückanzahl mit kleiner werdender Stückgröße erhöht (vgl. Abb. 3). Allerdings konnte ein derartiger Trend in Anlage $B$ und $\mathrm{C}$ nicht erkannt werden, was mit der geringeren Probenanzahl zusammenhängen kann. $\square \mathrm{ABS}$

QBioabbaubar Input 1

口HDPE

口HIPS

口LDPE

口PA

$\square \mathrm{PC/PBT}$

口PET

$\square P O M$

口PP

口PP/EDPM

口PS

口PU

口PVC

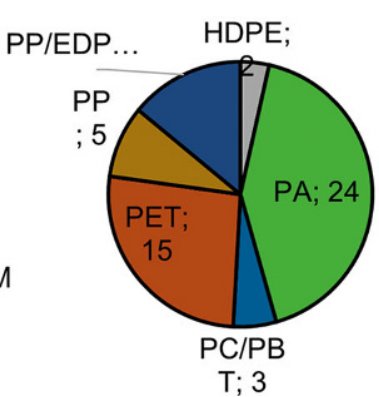

Abb. 5 Kunststoffarten in derRottegutfraktion $>10 \mathrm{~mm}$. Untersucht wurden Input 1 (November 2019), das daraus hergestellte Rottegut nach 10 Wochen Rottedauer vor dem Sieben („Vor Sieb“), sowie einem am Tag der Absiebung angelieferten Input 2 (Jänner 2020). Die Zahlen neben der jeweiligen Kunststoffart stellen die aufsummierten, absoluten Anzahlen der gefundenen Kunststoffpartikel der jeweiligen Kunststoffart dar, die in jeweils drei Proben gefunden wurden 


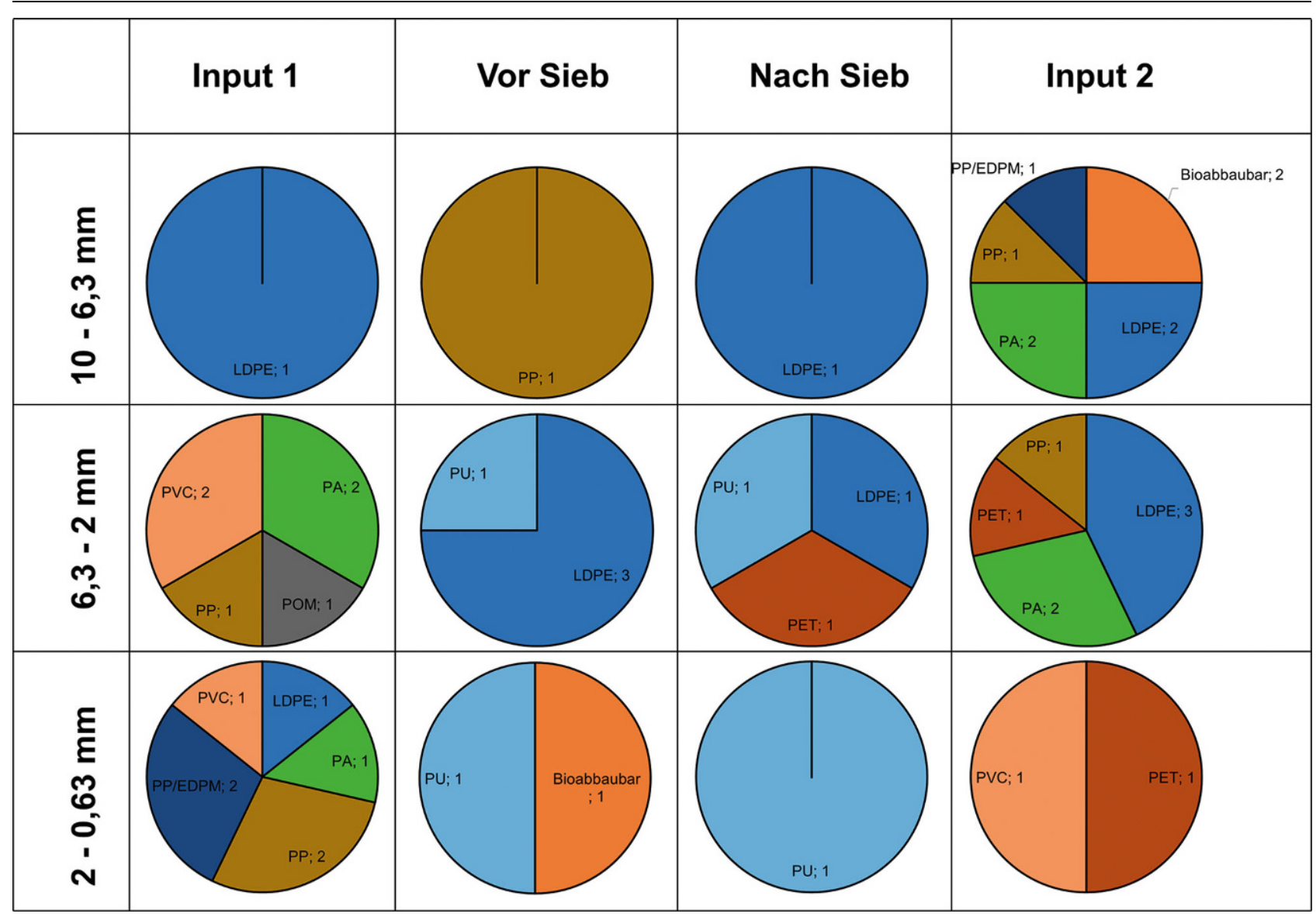

Abb. 6 Kunststoffarten in denFraktionen 10-6,3 mm (ersteZeile), 6,3-2 mm (zweiteZeile), 2-0,63 mm (3Zeile) von Input 1 (November 2019), vom daraus hergestellten Rottegut vor derWerksabsiebung („VorSieb“) und dem Kompost $<10$ mm(„NachSieb“), sowieeinem am Tag der Werksabsiebung angelieferten Input 2 (Jänner 2020). Die Zahlen neben der Kunststoffart stellen die absolute Anzahl der gefundenen Kunststoffpartikel der jeweiligen Kunststoffart dar, die in jeweils drei Proben gefunden wurden

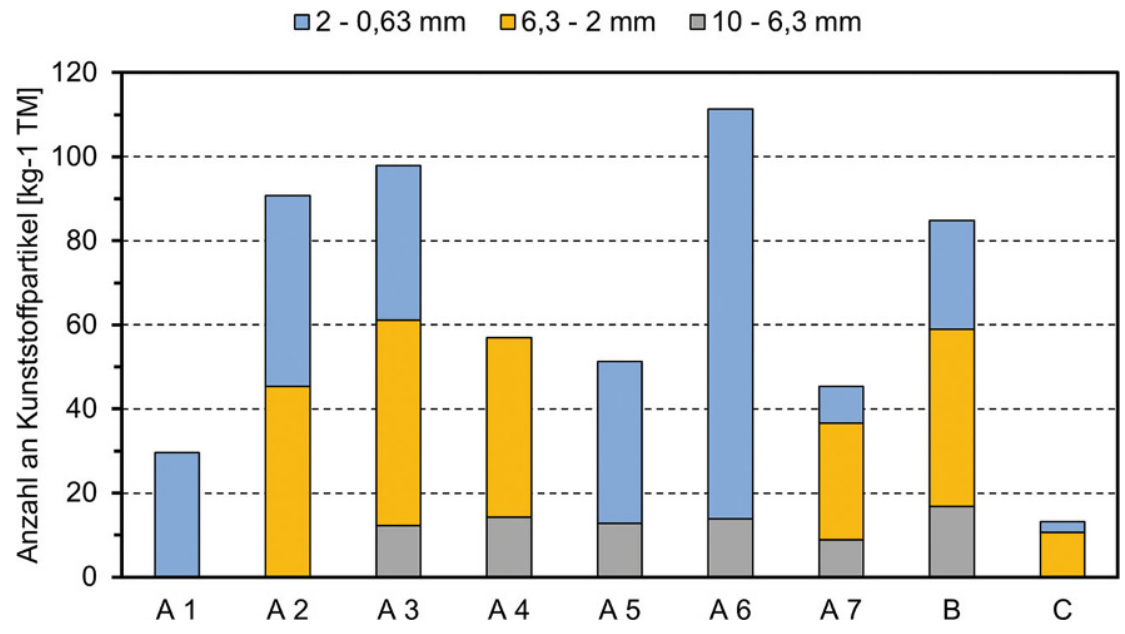

Abb. 7 Spezifische Kunststoffpartikelanzahlen (Anzahl prokg Trockenmasse) von sieben Komposten aus Anlage A (Kompost 1-7) und Komposten aus den Anlagen B und C. Bei Kompost 7 aus Anlage A handelt es sich um die oben beschriebene Probe „nach Sieb“. Bei den Daten Kompost 7, Anlage B und C handelt es sich jeweils um Mittelwerte aus drei Stichproben
Die Kunststoffpartikelmassen in den Komposten 1-7 lagen in einer Spannweite von $0-2,9 \mathrm{~g}$ pro $\mathrm{kg}$ TM (Abb. 8). Der Minimalwert von 0,0g kam durch die geringe Masse der Partikel im Kompost 1 zustande, die unter der Nachweisgrenze der verwendeten Waage blieben $(<0,1 \mathrm{mg})$. Die festgestellten Kunststoffkontaminationen entsprechen $0-0,29 \%$ TM; der Grenzwert der österreichischen Kompostverordnung (BMLFUW 2001) für eine Anwendung in der Landwirtschaft beträgt 0,2\% TM. 3 der 9 untersuchten Komposte wären demnach nicht für den Einsatz in der Landwirtschaft geeignet. Ein Einsatz dieser 3 Komposte in Landschaftsbau und Landschaftspflege sowie als Rekultivierungsschicht auf Deponien ist jedoch zulässig (Grenzwert $<0,4 \%$ TM). Der Mittelwert von $0,12 \%$ TM zeigt, dass im Durchschnitt die höchste Güteklasse $(0,2 \%$ eingehalten wird). Der Median von 0,06\% weist darauf hin, dass nur wenige, stärker belastete 
$\square 10-6,3 \mathrm{~mm} \quad \square 6,3-2 \mathrm{~mm} \quad \square 2-0,63 \mathrm{~mm}$

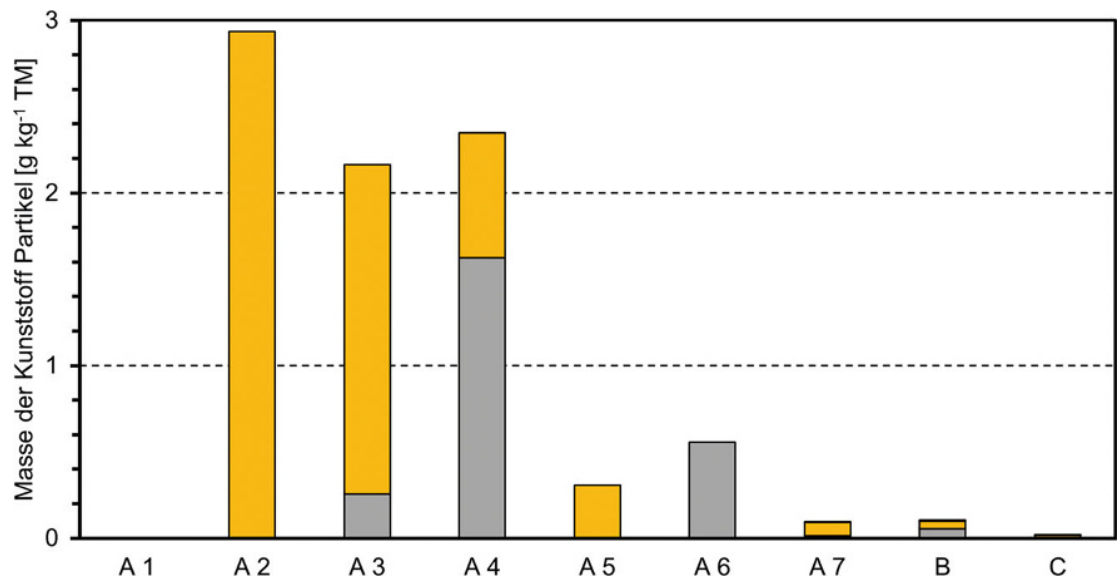

Abb. 8 Spezifische Kunststoffpartikelmassen (g pro kg Trockenmasse) von sieben Komposten aus Anlage $A$ (Kompost 1-7) sowie jeweils einem Kompost aus Anlagen $B$ und C. Dargestellt sind die anteiligen Kunststoffmassen der untersuchten Fraktionen. Im Kompost 1 lagen die Massen der Kunststoffpartikel unter der Nachweisgrenze der Waage $(<0,1 \mathrm{mg})$

Komposte für den höheren Mittelwert verantwortlich sind (vgl. Argumentation Partikelzahl).

Beurteilt über den Median unterschreitet zumindest die Hälfte der untersuchten Komposte auch den noch strengeren Grenzwert der Düngemittelverordnung von $0,1 \%$ Kunststoffkontamination (BMFLUW 2004).

Der Vergleich zwischen Partikelmassen und -anzahl zeigt, dass beide Größen nur eingeschränkt korrelieren. Der (lineare) Pearson-Korrelationskoeffizient $\rho$ ergibt Korrelationen von 0,44, 0,65 und 0,49 für die Fraktionen 10-6,3 mm, $6,3-2 \mathrm{~mm}$ und die Summe über alle Fraktionen, aber keine Korrelation für die kleinste untersuchte Fraktion von $2-0,63 \mathrm{~mm}(\rho=-0,16)$. Die schwache Korrelation von Partikelanzahl und -masse ist besonders gut erkennbar beim Vergleich der Komposte 2 und 6 aus Anlage A. Obwohl Kompost $620 \%$ mehr Partikel aufweist als Kompost 2, beträgt die Gesamtmasse der Partikel in Kompost 6 nur $18 \%$ von jener in Kompost 2. Kompost 6 weist allerdings hauptsächlich sehr kleine Partikel (Fraktion 2-0,63 mm) auf. Dieses Ergebnis zeigt, dass gerade bei Kunststoffmaterialien, die Variationen der Form (z. B. Folie vs. kompakter Partikel) zu starken Unterschieden zwischen Partikelzahlen und Massen führen kann.

In den 9 untersuchten Komposten wurden insgesamt 9 verschiedene Kunststoffarten gefunden, die sehr heterogen in den einzelnen Kornfraktionen verteilt sind. Tab. 3 stellt die gemittelten Daten der Anlagen dar. In Anlage A liegen dem Mittelwert 7 Komposte (einer davon aus 3 Stichproben), Anlage B 6 bzw. Anlage C 4 Stichproben jeweils eines Kompostes zugrunde.

In Anlage A enthielt die Fraktion 10-6,3 mm nur LDPE, während in der nächstfeineren Fraktion (6,3-2 mm) neben LDPE auch noch PVC, PP, PU und
PET gefunden wurden. Die Fraktion 2-0,63 $\mathrm{mm}$ war ebenso heterogen und enthielt LDPE, PP, PVC, PP/PE Blends, PU und PBT. Gesamt betrachtet, machten die Polyolefine PE/PP, LDPE und PP den größten Anteil von $70 \%$ aus, gefolgt von PVC (21\%), PU (5\%), PBT $(3 \%)$ und PET $(1,7 \%)$. Interessanterweise wurden keine biologisch abbaubaren Kunststoffe gefunden. Dies ist ein Indiz dafür, dass diese während einer Kompostierung nach Stand der Technik abgebaut bzw. in Partikel $<0,63 \mathrm{~mm}$ defragmentiert werden (nach EN 13432 zertifizierte biologisch abbaubare Werkstoffe müssen während der Kompostierung zu mindestens $90 \%$ in Partikel $<2 \mathrm{~mm}$ defragmentiert werden). Fraktionsabhängig wurden $52 \%$ der Partikel in der feinsten untersuchten Fraktion $(2-0,63 \mathrm{~mm})$ gefunden, gefolgt von $6,3-2 \mathrm{~mm}$ mit $38 \%$, während die gröbste Fraktion $10-6,3 \mathrm{~mm}$ nur $10 \%$ der gesamten Kunststoffbelastung aufweist. Das bedeutet, dass $90 \%$ der gefundenen Kunststoffpartikel Korndurchmesser zwischen 0,63 und 6,3 mm aufwiesen, und damit zum Mikrokunststoffbereich gezählt werden. Anzumerken ist, dass $52 \%$ der Partikel in der Fraktion $2-0,63 \mathrm{~mm}$ bei einer Untersuchung nach Kompost- bzw. Düngemittelverordnung nicht in die Bewertung der Kompostqualität berücksichtigt werden würden!

Der Kompost von Anlage B enthielt die heterogenste Kunststoffzusammensetzung in allen Fraktionen. In der Fraktion 10-6,3 mm finden sich LDPE, PP/PE, PU und HDPE. Die Fraktion 6,3-2 mm enthielt PP, PP/PE, PVC, HDPE und PA und die kleinste Fraktion (2-0,63 mm) enthielt LDPE, PP, PP/PE, PU, HDPE, PA und PET. Auch in Anlage B waren die Polyolefine (HDPE, LDPE, PP/PE und PP) zusammen mit $77 \%$ die häufigsten Polymere, während PA (10\%), PU (7\%), sowie PVC und

Tab. 3 Spezifische Partikelanzahl der jeweiligen Kunststoffart in 9 untersuchten Komposten der Anlagen A, B und C (Anzahl pro $\mathrm{kg}$ TM)

\begin{tabular}{|l|l|l|l|l|l|l|l|l|l|l|l|}
\hline \multirow{2}{*}{ Anlage A } & & LDPE & PP & PP/PE & PVC & PU & PBT & HDPE & PA & PET \\
& $10-6,3 \mathrm{~mm}$ & 7,6 & - & - & - & - & - & - & - & - \\
& $6,3-2 \mathrm{~mm}$ & 13,3 & 6,2 & 2,0 & 4,2 & 1,3 & - & - & - & 1,3 \\
\hline \multirow{3}{*}{ Anlage B } & $2-0,63 \mathrm{~mm}$ & 5,9 & 6,4 & 11,3 & 11,4 & 2,2 & 2,2 & - & - & - \\
& $10-6,3 \mathrm{~mm}$ & 2,8 & - & 2,8 & - & 2,8 & - & 8,4 & - & - \\
& $6,3-2 \mathrm{~mm}$ & - & 8,4 & 5,6 & 2,8 & - & - & 19,7 & 5,6 & - & \\
& $2-0,63 \mathrm{~mm}$ & 2,8 & 2,8 & 2,8 & - & 2,8 & - & 8,4 & 2,8 & 2,8 \\
\hline \multirow{2}{*}{ Anlage C } & $10-6,3 \mathrm{~mm}$ & - & - & - & - & - & - & - & - & - \\
& $6,3-2 \mathrm{~mm}$ & - & - & 2,6 & 5,3 & - & - & - & - & 2,6 \\
& $2-0,63 \mathrm{~mm}$ & - & - & - & - & - & - & - & - & 2,6 \\
\hline
\end{tabular}


PET (je $3 \%$ ) weit seltener vorkamen. In Fraktionen betrachtet, hatten $50 \%$ der Kunststoffe eine Größe zwischen 6,3 und $2 \mathrm{~mm}$. $30 \%$ der Kunststoffe wiesen Größen $2-0,63 \mathrm{~mm}$ auf und $20 \%$ lagen zwischen 10-6,3 mm.

Im Kompost der Anlage $\mathrm{C}$ wurden in der Fraktion 6,3-2 mm PVC, PP/PE sowie PET gefunden; in der Fraktion 2-0,63 mm fand sich nur noch PET.

\subsection{Vergleich mit Literaturdaten}

Der Literaturvergleich erfolgt mit Ergebnissen aus deutschen Kompostanlagen (Weithmann et al. 2018) bei denen Komposte - hergestellt aus unterschiedlichen Inputmaterialien und mit unterschiedlicher Rottetechnik untersucht wurden. In dieser Studie wurden Kunststoffpartikel im Größenbereich $10-1 \mathrm{~mm}$ erfasst. Diese werden mit den ausgewerteten Daten aus dieser Studie für Komposte im Größenbereich 10-0,63 mm verglichen. Durch die unterschiedlichen Maschenweiten ist ein Vergleich nur näherungsweise möglich. Da die Komposte aus dieser Studie auch kleinere Partikel erfassen, sind im Schnitt höhere Partikelanzahlen zu erwarten (Abb. 9).

Die von Weithmann et al. (2018) untersuchten Komposte (Bioabfall- und Grünschnittkomposte zwischen 8 und $15 \mathrm{~mm}$ Korngröße) stammen aus einer nicht näher beschriebenen Kompostanlage, in der jährlich $8000 \mathrm{t}$ Bioabfall zusammen mit $12.000 \mathrm{t}$ Grünschnitt verarbeitet werden. Das angelieferte Material wird durch ein 80-mmSieb fraktioniert, wobei die Fraktion $<80 \mathrm{~mm}$ nach einer Metallabtrennung direkt verwendet wird. Die Fraktion $>80 \mathrm{~mm}$ wird von Hand von Metallen, Steinen, Kunststoffen und Glas befreit und geschreddert und danach wieder mit der Fraktion $<80 \mathrm{~mm}$ vereinigt. Das aufbereitete Inputmaterial wird in einer geschlossenen Anlage kompostiert und erreicht dabei Rottetemperaturen $>70^{\circ} \mathrm{C}$. Nach einer nicht näher angeführten Zeitdauer wird das Rottegut für mehrere Monate in natürlich belüfteten Mieten gereift bzw. stabilisiert, bevor es bei 8 bzw. $15 \mathrm{~mm}$ abgesiebt und als Produkt zum Verkauf angeboten wird. Diese Komposte wiesen 20 (bei $8 \mathrm{~mm}$ ) bzw. 24 (bei $15 \mathrm{~mm}$ ) Kunststoffpartikel pro kg TM auf. Die Studie untersuchte auch Gärreste aus Biogasanlagen (ursprünglicher Input: Bioabfall aus Haushalten), wobei hier höhere Partikelzahlen (70 bis 122 pro $\mathrm{kg}$ TM) gefunden wurden als in den Komposten. Die niedrigste Anzahl an Kunststoffpartikeln $(0-11$ pro $\mathrm{kg}$ TM) wurde in landwirtschaftlichen Biogasanlagen gefunden, die höchste in Biogasanlagen, die gewerbliche Bioabfälle verwerten (896 pro $\mathrm{kg} \mathrm{TM}$ ). In Abb. 9 sind die von Weithmann et al. gefundenen Werte jeweils als horizontale Linien eingezeichnet. Alle untersuchten Komposte aus Anlage A ordnen sich zwischen den Vergleichskomposten aus „Haushalt und Grünschnitt gesiebt bei $15 \mathrm{~mm}$ “ und den Kompos- ten aus „Haushalt nach Anaerobbehandlung" ein und weisen im Mittel 69 (Median 57) Partikel pro kg TM auf. Vier Komposte (1, 4, 5 und 7) unterschreiten die Untergrenze der in Weithmann et al. (2018) untersuchten-Komposte „Haushalt nach anaerob“. Komposte aus Anlage B weisen mit 85 Partikel pro kg TM etwas höhere Kunststoffpartikelzahlen auf als der Durchschnitt von Anlage A, während Anlage C mit 13 Partikeln pro kg TM den niedrigsten Wert aufweist. Es ist aber anzumerken, dass alle Komposte der Anlagen A, B und $\mathrm{C}$ weniger als Faktor 5 streuen und daher vor allem in Anbetracht der starken Heterogenität der gefundenen Kunststoffe als sehr ähnlich belastet angesehen werden können.

Vergleichswerte für Kunststoffpartikel in Böden von Überschwemmungsgebieten der Schweiz zeigen, dass $30 \%$ der gefundenen Partikel im Größenbereich 5-0,5 mm und der Rest 0,5-0,125 mm lagen (Scheurer and Bigalke 2018). Gefunden wurden bis zu 593 Kunststoffpartikel pro kg TM im gesamten Größenbereich von 5-0,125 mm. In den Fraktionen $5-0,5 \mathrm{~mm}$ waren es 177 Kunststoffpartikel pro kg TM. Dieser Wert ist deutlich höher als die höchste Kontamination der in dieser Studie untersuchten Komposten. Da die Studie aus der Schweiz nur den Maximalwert genauer beschreibt, ist ein weiterer Vergleich nur eingeschränkt möglich. Der Mittelwert der Partikelanzahl wird für die 29 beprob-

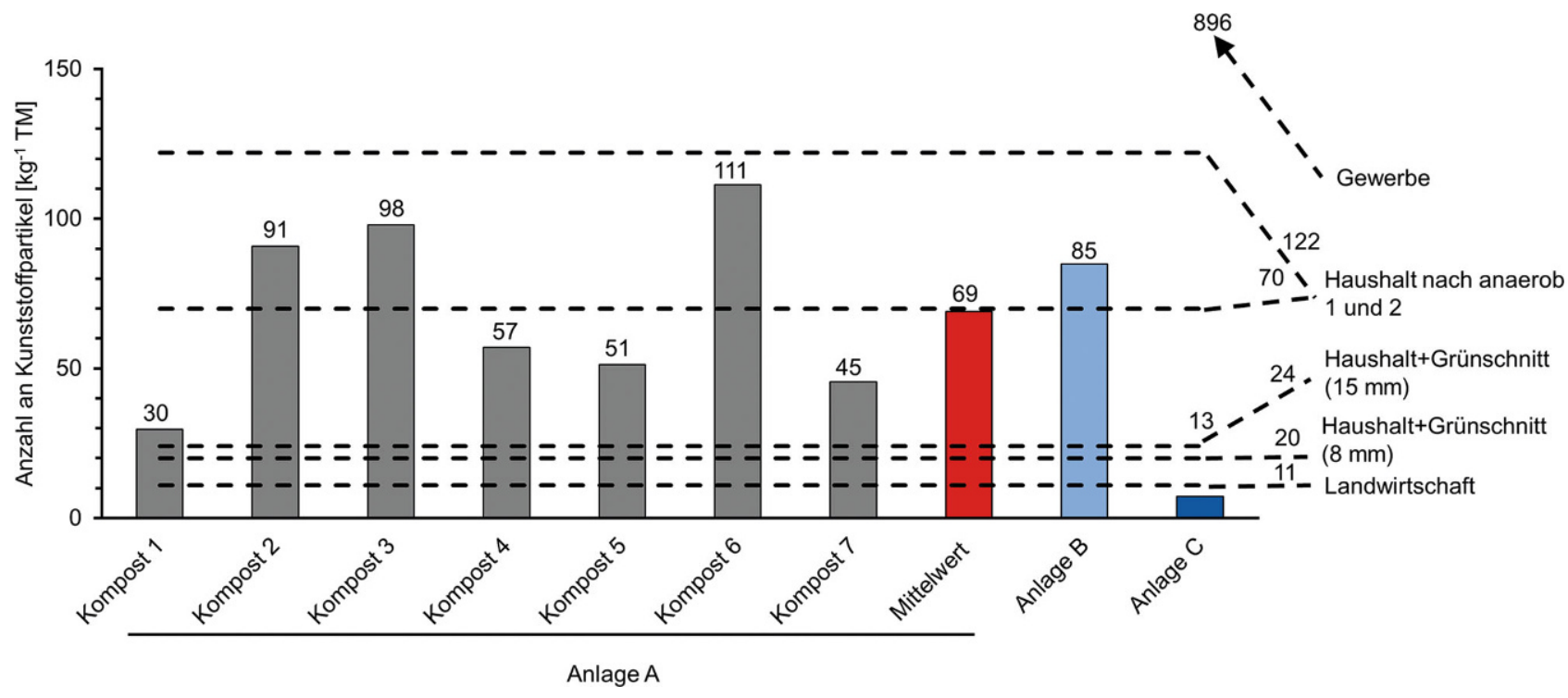

Abb. 9 Anzahl an Mikrokunststoffpartikeln 10-0,63 mm pro kg TM in 7 Komposten aus Anlage A (der Mittelwert ist in rot dargestellt) und je einem Kompost aus den Anlagen B (hellblau) und C (dunkelblau). Mit horizontalen Linien sind die von (Weithmann et al. 2018) angegebenen Mittelwerte an Kunststoffpartikeln >1 mm für diverse deutsche Komposte und Gärreste gekennzeichnet 
ten Orte mit ca. 120 Partikeln im Bereich $0,125-2,5 \mathrm{~mm}$ angegeben, wovon 36 Partikel $(30 \%)$ in einem Fraktionsbereich von ca. 2,5-0,5 mm liegen (siehe oben). Dieser Wert ist zwar um $50 \%$ geringer als der Mittelwert von Anlage A, aber immerhin fast dreimal höher als die Partikelanzahl in Anlage C. Das bedeutet, dass der hochwasserbedingte Austrag aus Flüssen zu ähnlich hohen Mengenbelastungen an Mikrokunststoffpartikeln in flussnahen Böden führt, wie in Bioabfallkomposten enthalten sind.

\section{Zusammenfassung}

Die Untersuchung von 9 Komposten aus drei österreichischen nach dem Stand der Technik betriebenen Kompostanlagen (offene Mietenkompostierung) ergab Kunststoffpartikelanzahlen im Bereich zwischen 13 und 111 Partikeln pro kg TM. Dies entspricht Konzentrationen von bis zu 2,9 $\mathrm{g}$ pro $\mathrm{kg} \mathrm{TM}$ im Größenbereich von $10-0,63 \mathrm{~mm}$. Im betrachteten Größenbereich wurden vor allem gängige Verpackungskunststoffe gefunden, von denen die Polyolefine LDPE, HDPE, PP und PP/PEBlends bis $\mathrm{zu} 77 \%$ der gefundenen Kunststoffpartikel ausmachten.

Bezüglich des Grenzwerts für Kunststoff erfüllten 6 von 9 der untersuchten Komposte sowohl die Kriterien für den Einsatz in der Landwirtschaft nach Kompostverordnung, als auch die strengeren Anforderungen der Düngemittelverordnung. Die anderen drei Komposte sind laut Kompostverordnung für den Einsatz in Landschaftsbau und Landschaftspflege sowie als Rekultivierungsschicht auf Deponien zulässig $(<0,4 \% \mathrm{TM})$.

Der Vergleich mit Komposten aus Deutschland zeigt, dass die in dieser Studie untersuchten Komposte ähnliche Kunststoffbelastungen (Weithmann et al. 2018) aufweisen. Gemäß einer Studie aus der Schweiz (Scheurer and Bigalke 2018) findet sich in Böden von einigen Überschwemmungsgebieten mehr Mikroplastik als in den hier untersuchten Komposten.

Die in dieser Studie gefundene Mikrokunststoffbelastung in Komposten lag zwischen 18 und 111 Partikeln pro kg TM bzw. hinsichtlich Masse bei 0,02 und 2,9 g pro kg TM. Bezogen auf die jährlich in Österreich hergestellte Kompostmenge von 339.200t (BAWP 2017), ergeben diese Zahlen zwischen 6 und 37 Mio. Mikrokunststoffpartikel bzw. eine Masse von 5-10t Kunststoff. Das entspricht $0,02-3,2 \%$ der jährlich in Österreich anfallenden Kunststoffverpackungsabfälle von 302.306t (BAWPStatusbericht 2020). Pro EinwohnerIn ergeben sich daraus für Österreich zwischen 0,6 und $111 \mathrm{~g}$ pro EW und Jahr an Kunststoffkontamination durch Komposte, während die Konsortialstudie für Deutschland (Bertling et al. 2018) $169 \mathrm{~g}$ pro EW und Jahr abschätzte. Die Konsortialstudie ging für Deutschland von einer Gesamtmikrokunststoffbelastung von $2880 \mathrm{~g}$ pro EW und Jahr aus und schätzte den Anteil der Kompostierung an der Gesamtbelastung auf $5,6 \%$ oder $169 \mathrm{~g}$ pro EW und Jahr. Unter der Annahme, dass die für Deutschland geschätzte jährliche Pro-Kopf-Menge an Mikrokunststoffen (2880 g pro EW und Jahr) auf Österreich übertragbar ist, würde der Anteil der Kompostausbringung an der Gesamtmikrokunststoffbelastung zwischen 0,02 und 3,9\% ausmachen.

In einer detailliert untersuchten Miete konnte festgestellt werden, dass sich die Kunststoffpartikelzahlen während des Rotteprozesses verändern. So spielt einerseits der Masseverlust des Rotteguts durch die Mineralisierung eine große Rolle (er führt zu einer relativen Erhöhung der Mikrokunststoffkonzentrationen), andererseits verteilt sich der Kunststoff durch Desintegration in kleinere Korngrößenfraktionen. Einen weiteren großen Einfluss auf die Kunststoffmenge stellt die Kompostendaufbereitung (Werksabsiebung) dar. Die gegenständliche Untersuchung ergab Hinweise darauf, dass neben Kunststoffpartikeln, die größer als der Sieblochdurchmesser sind, auch kleinere Kunststoffpartikel entfernt werden. Die wahrscheinlichste Erklärung für diese Beobachtung ist, dass kleinere Kunststoffpartikel an großen Matrixpartikeln anhaften und mit dem Siebüberlauf ausgeschleust werden. Wodurch diese Anhaftung beeinflusst wird, bleibt aufgrund der einmaligen Untersuchung vorerst unklar. Ein Einfluss des Wassergehalts ist wahrscheinlich. Dass durch eine Erhöhung des Wassergehalts vor der Absiebung aber tatsächlich die Mikrokunststoffbelastung in Komposten verringert werden kann, ist stark anzuzweifeln. Ein höherer Wassergehalt des Rotteguts bedeutet zwangsläufig auch eine Verringerung der Kompostausbeute (mit den abgetrennten Mikrokunststoffen landet zwangsläufig auch mehr Kompostfraktion im Siebüberlauf). Ferner konnte gezeigt werden, dass durch die Absiebung vor allem schwere Partikel aus dem Kompost entfernt wurden. Weiters gibt es Hinweise, dass auch jahreszeitlich bedingte Schwankungen auftreten.

Funding Open access funding provided by University of Natural Resources and Life Sciences Vienna (BOKU).

Open Access Dieser Artikel wird unter der Creative Commons Namensnennung 4.0 International Lizenz veröffentlicht, welche die Nutzung, Vervielfältigung, Bearbeitung, Verbreitung und Wiedergabe in jeglichem Medium und Format erlaubt, sofern Sie den/die ursprünglichen Autor(en) und die Quelle ordnungsgemäß nennen, einen Link zur Creative Commons Lizenz beifügen und angeben, ob Änderungen vorgenommen wurden.

Die in diesem Artikel enthaltenen Bilder und sonstiges Drittmaterial unterliegen ebenfalls der genannten Creative Commons Lizenz, sofern sich aus der Abbildungslegende nichts anderes ergibt. Sofern das betreffende Material nicht unter der genannten Creative Commons Lizenz steht und die betreffende Handlung nicht nach gesetzlichen Vorschriften erlaubt ist, ist für die oben aufgeführten Weiterverwendungen des Materials die Einwilligung des jeweiligen Rechteinhabers einzuholen.

Weitere Details zur Lizenz entnehmen Sie bitte der Lizenzinformation auf http://creativecommons.org/licenses/ by/4.0/deed.de. 
Ambrosini, R., Azzoni, R.S., Pittino, F., Diolaiuti, G., Franzetti, A., Parolini, M. (2019): First evidence of microplastic contamination in the supraglacial debris of an alpine glacier. Environ Pollut 253, 297-301.

Andrady, A.L. (2017): The plastic in microplastics: A review. Mar Pollut Bull 119, 12-22.

BAWP (2017): Bundesabfallwirtschaftsplan 2017 Teil 1. Bundesministerium für Nachhaltigkeit und Tourismus, Sektion V, Wien.

BAWP-Statusbericht (2020): Die Bestandsaufnahme der Abfallwirtschaft in Österreich-Statusbericht 2020. Bundesministerium für Klimaschutz, Umwelt, Energie, Mobilität, Innovation und Technologie, Abteilung V/3: Abfallwirtschaftsplanung, Abfallbehandlung und Altlastensanierung, Wien.

Bertling, J., Bertling, R., Hamann, L. (2018): Kunststoffe in der Umwelt: Mikro-und Makroplastik. Ursachen, Mengen, Umweltschicksale, Wirkungen, Lösungsansätze, Empfehlungen. Kurzfassung der Konsortialstudie, FraunhoferInstitut für Umwelt-, Sicherheit-und Enegietechnik UMSICHT (Hrsg.), Oberhausen.

BMFLUW (2004): Verordnung des Bundesministers für Land- und Forstwirtschaft, Umwelt und Wasserwirtschaft, mit der Bestimmungen zur Durchführung des Düngemittelgesetzes $1994 \mathrm{er}$ lassen werden (Düngemittelverordnung 2004), Bundesgesetzblatt für die Republik Österreich II Nr. 100/2004.

BMLFUW (2001): Verordnung des Bundesministers für Land- und Forstwirtschaft, Umwelt und Wasserwirtschaft über Qualitätsanforderungen an Komposte aus Abfällen (Kompostver- ordnung), Bundesgesetzblatt für die Republik Österreich II Nr. 292/2001.

Browne, M. A., Crump, P., Niven, S.J., Teuten, E., Tonkin, A., Galloway, T., Thompson, R. (2011): Accumulation of Microplastic on Shorelines Woldwide: Sources and Sinks. Environ Sci Technol 45, 9175-9179.

Cole, M., Lindeque, P., Halsband, C., Galloway, T.S. (2011): Microplastics as contaminants in the marine environment: A review. Mar Pollut Bull 62, 2588-2597.

Cox, K.D., Covernton, G.A., Davies, H.L., Dower, J.F., Juanes, F., Dudas, S.E. (2019): Human Consumption of Microplastics. Environ Sci Technol 53, 7068-7074.

Eerkes-Medrano, D., Thompson, R.C., Aldridge, D.C. (2015): Microplastics in freshwater systems: A review of the emerging threats, identification of knowledge gaps and prioritisation of research needs. Water Res 75, 63-82.

EFSA (2016): (EFSA Panel on Contaminants in the Food Chain) Statement on the presence of microplastics and nanoplastics in food, with particular focus on seafood. EFSA Journal 14, 1-30. Kenyon, K.W., Kridler, E. (1969): Laysan Albatrosses Swallow Indigestible Matter. Auk 86, 339-343.

Machado, A.A.D., Lau, C.W., Kloas, W., Bergmann, J., Bacheher, J.B., Faltin, E., Becker, R., Gorlich, A.S., Rillig, M.C. (2019): Microplastics Can Change Soil Properties and Affect Plant Performance. Environ Sci Technol 53, 6044-6052.

Machado, A.A.D., Lau, C.W., Till, J., Kloas, W., Lehmann, A., Becker, R., Rillig, M.C. (2018): Impacts of Microplastics on the Soil Biophy- sical Environment. Environ Sci Technol 52, 9656-9665.

Ng, E.L., Lwanga, E.H., Eldridge, S.M., Johnston, P., Hu, H.W., Geissen, V., Chen, D.L. (2018): An overview of microplastic and nanoplastic pollution in agroecosystems. Sci Total Environ 627, 1377-1388.

Qi, R.M., Jones, D.L., Li, Z., Liu, Q., Yan, C.R. (2020): Behavior of microplastics and plastic film residues in the soil environment: A critical review. Sci Total Environ 703. https://doi.org/10. 1016/j.scitotenv.2019.134722

Scheurer, M., Bigalke, M. (2018): Microplastics in Swiss Floodplain Soils. Environ Sci Technol 52, 3591-3598.

Schwabl, P., Koppel, S., Konigshofer, P., Bucsics, T., Trauner, M., Reiberger, T., Liebmann, B. (2019): Detection of Various Microplastics in Human Stool A Prospective Case Series. Ann Intern Med 171, 453-+.

Weithmann, N., Moller, J.N., Loder, M.G.J., Piehl, S., Laforsch, C., Freitag, R. (2018): Organic fertilizer as a vehicle for the entry of microplastic into the environment. Sci Adv 4 https://doi.org/10.1126/sciadv.aap8060

Hinweis des Verlags Der Verlag bleibt in Hinblick auf geografische Zuordnungen und Gebietsbezeichnungen in veröffentlichten Karten und Institutsadressen neutral. 